\title{
Desenvolvimento micelial de Lentinula edodes como efeito da profundidade e suplementação do substrato(1)
}

\author{
Ivan Henrique Rossi( ${ }^{(2)}$, Antonio Carlos Monteiro ${ }^{(2)}$ e José Octávio Machado ${ }^{(2)}$
}

\begin{abstract}
Resumo - Este trabalho objetivou avaliar a velocidade do desenvolvimento micelial de Lentinula edodes (Berk.) Pegler, o cogumelo Shiitake, como efeito da profundidade e da suplementação do substrato à base de bagaço de cana, com diferentes quantidades de farelo de arroz e melaço de cana. Foi instalado um experimento em esquema fatorial 7 × 2 (sete níveis de farelo ou melaço x duas fases de crescimento), utilizando frascos de vidro autoclaváveis para contenção dos substratos. As proporções de farelo de arroz testadas foram: $0,10,15,20,25,30$ e $40 \%$ (peso seco/peso seco de bagaço); as concentrações de melaço de cana foram: 0, 10, 20,30, 40, 50 e $60 \mathrm{~g} / \mathrm{kg}$ de substrato. Fitas de papel milimetrado aderidas externamente ao frasco serviram para medir o desenvolvimento do micélio. Para diferenciação das velocidades em função da profundidade, dividiu-se o crescimento em duas fases: inicial (metade superior do frasco) e final (metade inferior). A velocidade de miceliação na fase inicial foi sempre superior à da fase final, independentemente da quantidade de suplemento. Altas proporções de farelo diminuíram a velocidade de miceliação, principalmente na fase final, e o melaço de cana não influiu na rapidez do crescimento.
\end{abstract}

Termos para indexação: cogumelos, cana-de-açúcar, bagaço, melaço, arroz, farelo.

Mycelium production rate in Lentinula edodes as an effect of depth and supplementation of the substrate

\begin{abstract}
The objective of the present study was to assess the rate of mycelium development of Lentinula edodes (Berk.) Pegler as an effect of depth and supplementation of the sugar cane bagasse substrate with different amounts of rice bran and sugar cane molasses. The experimental design consisted in a $7 \times 2$ factorial scheme (seven levels of bran or molasses $\mathrm{x}$ two growth phases) using autoclavable glass flasks to keep the substrates. The proportions of rice bran tested were: $0,10,15,20,25,30$ and $40 \%$ (dry weight/bagasse dry weight), and the concentrations of sugar cane molasses were: $0,10,20,30,40$, 50 and $60 \mathrm{~g} / \mathrm{kg}$ substrate. Graph paper strips externally sticked to the flask were used to measure the mycelial development. To differentiate the growth as a function of depth, the mycelial development was divided into two phases: an initial one (upper half of the flask) and a final one (lower half). The rate of mycelium formation was always higher in the early growth than in the final phase regardless of the amount of supplement. High bran proportions reduced the rate of mycelium formation, especially during the final phase, and sugar cane molasses did not affect growth rate.
\end{abstract}

Index terms: fungi, sugar cane, bagasse, molasses, bran, rice.

(1) Aceito para publicação em 28 de agosto de 2000

Extraído da dissertação de mestrado apresentada pelo primeiro autor à Faculdade de Ciências Agrárias e Veterinárias (FCAV), Universidade Estadual Paulista (UNESP), Jaboticabal, SP. Parcialmente financiado pela FAPESP.

(2)UNESP, FCAV, Dep. de Produção Vegetal, Via de Acesso Prof. Paulo Donato Castellane, s/n, CEP 14884-900 Jaboticabal, SP. E-mail: montecar@fcav.unesp.br

\section{Introdução}

A produção de Shiitake, Lentinula edotes (Berk.) Pegler, um dos cogumelos mais consumidos no mundo (Royse, 1985; Rajarathnam et al., 1992), vem aumentando nas últimas décadas, com expectativa de continuar em ascensão (Worral \& Yang, 1992). No Brasil, seu cultivo vem crescendo significativa- 
mente, devido ao bom retorno econômico, possibilidade de ser cultivado em pequenas áreas e necessitar de baixo investimento inicial (Rossi, 1999).

Tradicionalmente, o Shiitake é produzido em cepos de eucalipto, mas o cultivo axênico em substratos sintéticos à base de diversos resíduos agrícolas vem ganhando espaço na produção comercial deste cogumelo, uma vez que a colheita acontece mais rapidamente e a eficiência biológica do fungo é bastante elevada (Przybylowicz \& Donoghue, 1988; Hiromoto, 1991; Levanon et al., 1993).

Por ser apto a utilizar lignina, celulose e hemicelulose como fonte de carbono e nutrientes, o Shiitake pode ser cultivado em uma grande variedade de resíduos agrícolas, dentre eles o bagaço de cana (Buswell et al., 1996; Eira \& Minhoni, 1996; Rossi, 1999). Por ter característica fibrosa, quando prensado, o bagaço pode condicionar espaços com aeração suficiente para o crescimento micelial, uma vantagem para o cultivo de Shiitake. No entanto, por ser resultante de cana-de-açúcar que sofreu diversos tipos de tratamento e lavagens em usinas de produção de álcool e açúcar, é um resíduo final rico em parede celular, de baixo conteúdo de células inteiras, baixa densidade e pobre em proteínas e minerais (Santos, 1990). Com isso, a suplementação com nutrientes, de preferência solúveis para estarem prontamente disponíveis para o fungo, é necessária.

Uma fonte de carbono e nutrientes, que pode ser utilizada para cultivo de $L$. edodes em substratos à base de bagaço, é o melaço de cana (Rossi, 1999). Song et al. (1987) mostram que solução aquosa de melaço na concentração de 30,0 g/L é capaz de manter um crescimento micelial vigoroso, o que sugere que este suplemento pode sustentar miceliação rápida de $L$. edodes em bagaço de cana. Segundo Zanetti \& Ranal (1997), fontes de carbono, como glicose e frutose, são importantes fornecedoras de energia para a atividade metabólica dos cogumelos, e constituem a base para síntese de diversas moléculas que resultam em aproximadamente $50 \%$ do peso da matéria seca dos corpos de frutificação, em carbono.

Outra fonte de nutrientes utilizada para suplementar o bagaço de cana é o farelo de arroz (Eira \& Minhoni, 1996; Iizuka, 1997; Rossi, 1999). Segundo vários autores, o farelo estimula o crescimento micelial de diversas espécies de cogumelos, promovendo, assim, a rápida colonização do substrato (Fasidi \& Kadiri, 1993; Royse ,1996; Teixeira, 1996).
A utilização dos diversos tipos de substratos pelo fungo depende da sua capacidade de secretar celulases, hemicelulases e ligninases, liberando nutrientes para seu crescimento (Buswell et al., 1996). As trocas gasosas dos substratos, durante o crescimento vegetativo, são bastante importantes para a produção destas enzimas. Segundo Donoghue \& Denison (1995), o $\mathrm{O}_{2}$ estimula o crescimento do fungo, e altas concentrações de $\mathrm{CO}_{2}$ afetam a sua atividade enzimática, diminuindo, assim, a velocidade de miceliação. Cultivando L. edodes em substratos com diferentes diâmetros de partícula de serragem, Donoghue \& Denison (1996) encontraram menores níveis de $\mathrm{CO}_{2}$ e maiores de $\mathrm{O}_{2}$ quando as partículas eram maiores, possibilitando, dessa forma, melhor troca gasosa. Trocas gasosas deficientes durante o crescimento do fungo podem diminuir a velocidade de miceliação, em virtude da inibição da atividade enzimática.

A velocidade de miceliação pode ser alterada à medida que o fungo se aprofunda no substrato. Considerando que a suplementação pode modificar a estrutura do substrato, dificultando as trocas gasosas, é possível que isso tenha implicação sobre a velocidade de miceliação do fungo, como efeito da profundidade do substrato.

Este trabalho objetivou avaliar a velocidade do desenvolvimento micelial de $L$. edodes como efeito da profundidade do frasco utilizado para acondicionar o substrato à base de bagaço de cana e da suplementação com farelo de arroz e melaço de cana.

\section{Material e Métodos}

Foi utilizada uma cultura do isolado JAB-K1, crescida em meio mínimo (Pontecorvo et al., 1953), a partir de basidiocarpo de $L$. edodes, obtido de produtores da região noroeste do Estado de São Paulo (Teixeira, 1996). O isolado é mantido em cultura-estoque na coleção do Laboratório de Microbiologia da Faculdade de Ciências Agrárias e Veterinárias, UNESP, Jaboticabal, SP. A partir desta cultura, obteve-se a semente-inóculo utilizada no experimento, constituída de $L$. edodes crescido em bagaço de cana enriquecido com $25 \%$ de farelo de arroz e $30 \mathrm{~g}$ de melaço de cana/kg de substrato (Rossi, 1999).

O substrato foi elaborado com bagaço de cana suplementado com farelo de arroz não desengordurado, melaço de cana, e misturas dos dois suplementos, com- 
pondo as variáveis do experimento. As quantidades testadas de cada suplemento foram: $0,10,15,20,25,30$ e $40 \%$ de farelo de arroz (peso seco/peso seco de bagaço), e 0 , $10,20,30,40,50$ e $60 \mathrm{~g}$ de melaço de cana/kg de substrato. Adicionou-se água para corrigir a umidade do substrato a $50 \%$. O farelo foi misturado ao bagaço antes da elevação da umidade, e o melaço, diluído na água de correção.

Para contenção dos substratos, foram utilizados frascos autoclaváveis de vidro, cilíndricos, medindo externamente $120 \mathrm{~mm}$ de altura e $62 \mathrm{~mm}$ de diâmetro. Os diferentes substratos foram distribuídos sem determinação de peso, mas com mesma altura em cada frasco, e com grau semelhante de compactação, sendo autoclavados a $121^{\circ} \mathrm{C}$ durante 90 minutos. Cada frasco correspondeu a uma repetição e o número de repetições por tratamento variou de 3 a 6 , porque foram eliminados os frascos que apresentaram a menor suspeita de contaminação.

Após a inoculação, os frascos foram colocados em local com temperatura entre $25^{\circ} \mathrm{C}$ e $28^{\circ} \mathrm{C}$ e ausência de iluminação, para o crescimento micelial. Os frascos receberam quatro fitas de papel milimetrado, verticalmente, em posições opostas, as quais serviram como instrumento de medida do crescimento do micélio em direção ao fundo do frasco. As medidas foram anotadas em milímetros percorridos pelo micélio sob as fitas na região onde o vidro apresentava diâmetro uniforme, que correspondeu aproximadamente a $70 \mathrm{~mm}$ de comprimento (Rossi, 1999).

Para verificação da velocidade de miceliação, foi avaliado o tempo gasto para o fungo atingir a metade da distância máxima percorrida pelo micélio durante o tempo total de crescimento (inicial) e o tempo necessário para percorrer o restante (final). Dessa forma, obtiveram-se duas velocidades de miceliação: a inicial, mais próxima; e a final, mais distante da superfície do substrato.

As análises dos resultados foram feitas utilizando um esquema fatorial $7 \times 2$ (sete proporções de farelo ou sete concentrações de melaço x duas fases de crescimento). $\mathrm{O}$ delineamento experimental utilizado foi o inteiramente casualizado (DIC).

\section{Resultados e Discussão}

Observa-se que nas duas fases de crescimento, à medida que aumenta a proporção de farelo, diminui a velocidade de miceliação (Tabela 1). Devido ao fato de o suplemento ter apresentado baixa relação $\mathrm{C}: \mathrm{N}$ (18:1) em comparação com o bagaço de cana (107:1), quando adicionado em altas quantidades proporciona um substrato com relação estreita, podendo reduzir a velocidade de miceliação de $L$. edodes, devido à alta concentração de $\mathrm{N}$, que limita a degradação da
Tabela 1. Velocidade de miceliação de Lentinula edodes (mm/dia) em razão da suplementação do substrato com farelo de arroz e com melaço de cana, em cada fase de crescimento $^{(1)}$.

\begin{tabular}{ccccc}
\hline \multirow{2}{*}{ Suplemento } & \multicolumn{2}{c}{ Fase de crescimento } & \multirow{2}{*}{$\begin{array}{c}\text { Teste } \\
\text { F }\end{array}$} & DMS \\
\cline { 2 - 3 } & Inicial & \multicolumn{2}{c}{ Final } & \\
\cline { 1 - 3 } Farelo $(\%)$ & & & & \\
0 & $1,71 \mathrm{aA}$ & $1,47 \mathrm{aB}$ & $42,5^{* *}$ & 0,07 \\
10 & $1,79 \mathrm{aA}$ & $1,34 \mathrm{bB}$ & $32,5^{* *}$ & 0,15 \\
15 & $1,70 \mathrm{aA}$ & $1,22 \mathrm{cB}$ & $48,4^{* *}$ & 0,14 \\
20 & $1,62 \mathrm{abA}$ & $1,08 \mathrm{~dB}$ & $46,4^{* *}$ & 0,18 \\
25 & $1,43 \mathrm{bcA}$ & $1,04 \mathrm{~dB}$ & $113,7^{* *}$ & 0,07 \\
30 & $1,35 \mathrm{cdA}$ & $0,99 \mathrm{~dB}$ & $124,0^{* *}$ & 0,06 \\
40 & $1,22 \mathrm{dA}$ & $0,74 \mathrm{eB}$ & $83,6^{* *}$ & 0,10 \\
\hline Teste F & $21,04 * *$ & $90,87 * *$ & - & - \\
DMS & 0,25 & 0,14 & - & - \\
\hline Melaço $(\mathrm{g} / \mathrm{kg})$ & & & & \\
0 & $1,46 \mathrm{aA}$ & $1,16 \mathrm{abB}$ & $50,3^{* *}$ & 0,09 \\
10 & $1,61 \mathrm{aA}$ & $1,15 \mathrm{abB}$ & $61,9^{* *}$ & 0,12 \\
20 & $1,51 \mathrm{aA}$ & $1,15 \mathrm{abB}$ & $73,0^{* *}$ & 0,09 \\
30 & $1,63 \mathrm{aA}$ & $1,12 \mathrm{abB}$ & $60,4^{* *}$ & 0,13 \\
40 & $1,47 \mathrm{aA}$ & $1,17 \mathrm{aB}$ & $42,3^{* *}$ & 0,10 \\
50 & $1,57 \mathrm{aA}$ & $1,09 \mathrm{abB}$ & $68,8^{* *}$ & 0,11 \\
60 & $1,55 \mathrm{aA}$ & $1,05 \mathrm{bB}$ & $147,4^{* *}$ & 0,08 \\
\hline Teste F & $1,97^{\mathrm{ns}}$ & $2,77 *$ & - & - \\
DMS & 0,20 & 0,11 & - & - \\
\hline
\end{tabular}

(1)Médias seguidas de mesma letra, minúscula nas colunas e maiúscula nas linhas, não diferem entre si pelo teste de Tukey a $5 \%$ de probabilidade ns Não-significativo. * e**Significativo a $5 \%$ e a $1 \%$ de probabilidade, respectivamente.

lignina presente no bagaço. Neste contexto, Singh \& Verma (1996) mostraram que relação C:N baixa limitava o crescimento de L. lateritia. Kamra \& Zadrazil (1988), citados por Maziero (1990), admitiram ocorrer inibição da degradação da lignina em decorrência da alta concentração de $\mathrm{N}$ no substrato.

Este efeito de queda da velocidade, pela suplementação com farelo de arroz, em altas proporções, provavelmente se deve também ao fato de que, aumentando a quantidade de farelo em relação ao bagaço, altera-se a estrutura do substrato, ou seja, este fica menos fibroso e, quando prensado, forma menor quantidade de bolsas de ar. Com isso, a capacidade de troca gasosa do substrato é reduzida e, conseqüentemente, a concentração de $\mathrm{O}_{2}$ no seu interior é limitada. Esse efeito é melhor observado na fase final do crescimento (mais profunda), em que as menores proporções adotadas ( 0 a $15 \%$ ) diferiram entre si e foram superiores às demais. Nesta profundidade, o fornecimento de $\mathrm{O}_{2}$ oriundo do ambiente é 
menor e, dessa forma, o fungo praticamente depende do $\mathrm{O}_{2}$ inicialmente contido no substrato.

Comparando a velocidade entre as duas fases de crescimento, dentro de cada proporção de farelo de arroz (Tabela 1), observa-se que na fase inicial ela foi significativamente superior, quando adicionada qualquer quantidade do suplemento. Isto evidencia que, mais próximo à superfície do substrato, ocorre maior troca gasosa, permitindo maior fornecimento de $\mathrm{O}_{2}$, maior atividade lignocelulolítica e, conseqüentemente, rápido crescimento. Neste contexto, Donoghue \& Denison (1995) sugeriram que altos teores de $\mathrm{O}_{2}$ e $\mathrm{CO}_{2}$ podem estimular ou inibir, respectivamente, o crescimento micelial. Em trabalho subseqüente, Donoghue \& Denison (1996) mostraram que substratos com partículas maiores apresentaram teores mais elevados de $\mathrm{O}_{2}$ e menores de $\mathrm{CO}_{2}$. Leatham (1986) mostrou a importância do fornecimento de $\mathrm{O}_{2}$ durante o crescimento micelial, ao obter maior atividade de enzimas lignocelulolíticas nos primeiros 20 dias de cultivo, período em que a concentração desse elemento é maior. Leatham \& Stahmann (1987) observaram que a troca gasosa inadequada é fator inibitório para o crescimento micelial de L. edodes.

O melaço de cana não influiu na velocidade de miceliação de $L$. edodes em praticamente nenhuma das fases de crescimento (Tabela 1). Por outro lado, Song et al. (1987) obtiveram melhor crescimento micelial de $L$. edodes ao adicionarem $30 \mathrm{~g}$ de melaço/L de solução, e Leatham (1986) assumiu haver necessidade de fornecimento de açúcar para aumentar a atividade lignocelulolítica. Ao avaliar a velocidade em resposta à profundidade, observa-se que em todas as concentrações do suplemento, o micélio de L. edodes se desenvolveu mais rapidamente na fase inicial que na final. Isso se deve provavelmente ao fato de que, quanto mais próximo à superfície do substrato, maior é o fornecimento de $\mathrm{O}_{2}$, e quanto mais distante, maior é a limitação desse elemento, essencial para o desenvolvimento de $L$. edodes.

\section{Conclusões}

1. A suplementação do bagaço de cana com farelo de arroz nas maiores proporções diminui a velocidade de miceliação de $L$. edodes, principalmente na parte mais profunda do substrato.
2. O melaço de cana, como suplemento do bagaço de cana, não altera a velocidade de miceliação de L. edodes.

3. O micélio de L. edodes cresce mais rapidamente em bagaço de cana suplementado com farelo de arroz e melaço de cana quando está mais próximo à superfície do substrato, local onde há fornecimento de oxigênio.

\section{Referências}

BUSWELL, J. A.; CAI, Y. J.; CHANG, S. T. Ligninolytic enzyme production and secretion in edible mushroom fungi. In: INTERNATIONAL CONFERENCE ON MUSHROOM BIOLOGY AND MUSHROOM PRODUCTS, 2., 1996, University Park. Proceedings... University Park : Pennsylvania State University, 1996. p. 113-122.

Donoghue, J. D.; Denison, W. C. Commercial production of shiitake (Lentinula edodes) using whole$\log$ chip of Quercus, Lithocarpus, and Acer. In: INTERNATIONAL CONFERENCE ON MUSHROOM BIOLOGY AND MUSHROOM PRODUCTS 2., 1996, University Park. Proceedings... University Park : Pennsylvania State University, 1996. p. 265-275.

DOnOGHuE, J. D.; DENISON, W. C. Shiitake cultivation: gas phase during incubation influences productivity. Mycologia, New York, v. 87, n. 2, p. 239244, 1995.

EIRA, A. F.; MINHONI, M. T. A. Manual teórico-prático do cultivo de cogumelos comestíveis. Botucatu : Fundação de Estudos e Pesquisas Agrícolas e Florestais, 1996. $96 \mathrm{p}$.

FASIDI, I. O.; KADIRI, M. Use of agricultural wastes for the cultivation of Lentinus subnudus (Polyporales: Polyporaceae) in Nigeria. Revista de Biología Tropical, San José, v. 41, n. 3, p. 411-415, 1993.

HIROMOTO, B. T. Comparative analysis of shiitake culture systems. In: INTERNATIONAL CONGRESS ON THE SCIENCE AND CULTIVATION OF EDIBLE FUNGI, 13., 1991, Dublin. Proceedings... Dublin : Balkema, 1991. v. 2, p. 489-496.

IIZUKA, H. Production of Lentinus edodes mycelia extracts (LEM). Food Reviews International, New York, v. 13 , n. 3, p. 343-348, 1997. 
LEATHAM, G. F. The lignolytic activities of Lentinus edodes and Phanerochaete chrysosporium. Applied Microbiology and Biotechnology, Berlin, v. 24, p. 51-58, 1986.

LEATHAM, G. F.; STAHMANN, M. A. Effect of light and aeration on fruiting of Lentinula edodes. Transactions of the British Mycological Society, Cambridge (Inglaterra), v. 88, n. 1, p. 9-20, 1987.

LEVANON, D.; ROTHSCHILD, N.; DANAI, O.; MASAPHY, S. Bulk treatment of substrate for the cultivation of shiitake mushrooms (Lentinula edodes) on straw. Bioresource Technology, Oxford, v. 45, p. 63-64, 1993.

MAZIERO, R. Substratos alternativos para o cultivo de Pleurotus spp. São Paulo : USP, 1990. 136 p. Dissertação de Mestrado.

PONTECORVO, G.; ROPER, J. A.; HEMMONS, L. M.; McDONALD, K. D.; BUFTON, A. W. J. The genetics of Aspergillus nidulans. Advances in Genetics, San Diego, v. 5, p. 141-238, 1953.

PRZYBYLOWICZ, P.; DONOGHUE, J. Shiitake growers handbook: the art and science of mushroom cultivation. Dubuque : Kendall/Hunt, 1988. 217 p.

RAJARATHNAM, S.; SHASHIREKA, M. N.; BANO, Z. Biopotentialities of the basidiomacromycetes. Advances in Applied Microbiology, San Diego, v. 37, p. 233-361, 1992.

ROSSI, I. H. Suplementação de bagaço de cana para cultivo axênico do cogumelo Shiitake [Lentinula edodes (Berk.) Pegler]. Jaboticabal : UNESP, 1999. 120 p. Dissertação de Mestrado.
ROYSE, D. J. Effect of spawn run time and substrate nutrition on yield and a size of the shiitake mushroom Mycologia, New York, v. 77, n. 5, p. 756-762, 1985.

ROYSE, D. J. Specialty mushrooms. In: JANICK, J. (Ed.). Progress in new crops. Arlington : American Society for Horticultural Science, 1996. p. 464-475.

SANTOS, F. A. P. O bagaço de cana-de-açúcar tratado sob pressão de vapor como alternativa para a alimentação de bovinos na entressafra das pastagens. Campinas : Sociedade Brasileira de Zootecnia, 1990. 203 p.

SINGH, T. G.; VERMA, R. N. Studies on carbon and nitrogen of Lentinula lateritia (Berk.) Pegler strains from northeastern India. In: INTERNATIONAL CONFERENCE ON MUSHROOM BIOLOGY AND MUSHROOM PRODUCTS, 1996, University Park. Proceedings... University Park : Pennsylvania State University, 1996. p. 345-354.

SONG C. H.; CHO, K. Y.; NAIR, N. G. A synthetic medium for the production of submerged cultures of Lentinus edodes. Mycologia, New York, v. 79, n. 6, p. 866-876, 1987.

TEIXEIRA, E. M. Efeito da suplementação de serragem de Eucalyptus grandis (Hill ex Maiden), na velocidade e intensidade de colonização do substrato para produção de "Semente" de Letinula edodes (Berk.) Pegler e sua eficiência na produtividade. Jaboticabal : UNESP, 1996. 37 p. Dissertação de Mestrado.

WORRAL, J. J.; YANG, C. S. Shiitake and oyster mushroom production on apple pomace and sawdust. HortScience, Alexandria, v. 27, n. 10, p.1131-1133, 1992.

ZANETTI, A. L.; RANAL, M. A. Suplementação de canade-açúcar com guandu no cultivo de Pleurotus sp. 'Florida'. Pesquisa Agropecuária Brasileira, Brasília, v. 32, n. 9, p. 959-964, set. 1997. 